\title{
El espacio público en la ciudad popular: la vida entre laderas
}

L'espace public dans la ville populaire: la vie au sein de versants escarpés Public space in the rural city: life between the hillsides

\section{Franklin Alberto Velarde Herz}

\section{(2) OpenEdition}

\section{Journals}

Edición electrónica

URL: https://journals.openedition.org/bifea/8851

DOI: $10.4000 /$ bifea.8851

ISSN: 2076-5827

Editor

Institut Français d'Études Andines

Edición impresa

Fecha de publicación: 8 diciembre 2017

Paginación: 471-488

ISSN: 0303-7495

Referencia electrónica

Franklin Alberto Velarde Herz, «El espacio público en la ciudad popular: la vida entre laderas», Bulletin de l'Institut français d'études andines [En línea], 46 (3) | 2017, Publicado el 08 décembre 2017,

consultado el 28 juin 2022. URL: http://journals.openedition.org/bifea/8851 ; DOI: https://doi.org/ 10.4000/bifea.8851

\section{(c) $(1)$}

Les contenus du Bulletin de l'Institut français d'études andines sont mis à disposition selon les termes de la licence Creative Commons Attribution - Pas d'Utilisation Commerciale - Pas de Modification 4.0 International. 


\title{
El espacio público en la ciudad popular: la vida entre laderas
}

\author{
Franklin Alberto Velarde Herz
}

\begin{abstract}
Resumen
El espacio público se produce y transforma como parte del tejido físico y social del hábitat barrial. En la ciudad popular, el habitar barrial tiene efectos particulares sobre los usos del suelo, los procesos de consolidación, la organización de las personas y el paisaje urbano. Entonces, es pertinente preguntarnos: ¿Cómo se dan las formas de producción y apropiación de los espacios públicos dentro del hábitat popular? Y a partir de esto, ¿qué tipos de espacios públicos se producen como parte de los diferentes procesos de urbanización popular? Estas preguntas buscarán ser respondidas, teniendo como base un estudio de caso realizado en la ciudad de Lima, donde gran parte de los procesos de urbanización se han producido a partir de lotizaciones difusas e informales en zona de laderas. Esto ha representado un nuevo escenario geográfico para la producción y la consolidación de diversos espacios colectivos, lo cual nos permite repensar urbanísticamente lo público.
\end{abstract}

Palabras clave: urbanismo, espacio público, laderas, ciudad popular, urbanización popular, hábitat popular, producción del espacio, Lima

\section{L'espace public dans la ville populaire: la vie au sein de versants escarpés}

\section{Résumé}

L'espace public est produit et transformé dans le cadre du tissu physique et social de l'habitat du quartier. Dans la ville populaire, les modes d'habiter le quartier ont des effets particulières sur l'usage du sol, les processus de consolidation, l'organisation des personnes et le paysage urbain. Il est donc pertinent de se demander : Comment se définissent les formes de production et d'appropriation des espaces publics dans l'habitat populaire ? Et à partir de là, quels types d'espaces publics sont produits dans le cadre des différents processus d'urbanisation populaire ? Nous essaierons de répondre à ces questions à partir d'une étude de cas menée dans la ville de Lima, où une grande partie des processus

* Docente de la Facultad de Arquitectura y Urbanismo de la Pontificia Universidad Católica del Perú y miembro del Grupo de Investigación en Urbanismo, Gobernanza y Vivienda Social (CONURBPUCP), Av. Universitaria 1801, San Miguel, Lima-Perú. E-mail: velardeherz@gmail.com 
d'urbanisation s'est faite à partir d'occupations du sol diffuses et informelles au sein des versants escarpés. Cela a représenté un nouveau scénario géographique pour la production et la consolidation de divers espaces collectifs, ce qui nous permet de repenser l'espace public sur le plain urbanistique.

Mots-clés: urbanisme, espace public, versants escarpés, ville populaire, urbanisation populaire, habitat populaire, production de l'espace, Lima

\title{
Public space in the rural city: life between the hillsides
}

\begin{abstract}
Public space develops and stems from the physical and social fabric of the neighborhood habitat. Within the popular city, neighborhood habitat has particular effects on land use, consolidation processes, people organization, and urban landscape. Then, it is pertinent to ask ourselves: How do the forms of production and appropriation of public spaces within the popular habitat happen? And following this logic, what kind of public spaces arise from the different processes of popular urbanization? These questions will be addressed, based on a case study investigation conducted in the city of Lima, a city where a large part of the urbanization processes have originated from diffuse and informal land distribution in the hillside area. This phenomenon represents a new geographical landscape for the production and consolidation of various collective spaces, which allows us to rethink the public spaces from an urban perspective.
\end{abstract}

Keywords: urbanism, public space, hillsides, popular city, popular urbanization, popular habitat, production of space, Lima

El espacio público como forma urbana se produce y transforma, interactuando como componente del tejido físico y a través de las diversas formas de habitar que se despliegan y superponen sobre un territorio. Es decir, existe una relación cercana entre las formas de urbanización, los procesos de consolidación barrial y los distintos entornos geográficos con las características físicas y los usos de los espacios públicos. Asimismo, la ciudad popular como paradigma urbano nos ofrece características particulares en cuanto a los usos del suelo, los procesos de consolidación que dan forma al paisaje urbano y a la organización de las personas. Por lo tanto, es interesante preguntarnos: ¿Cómo se dan las formas de producción y apropiación de los espacios públicos dentro del hábitat popular?

Para poder entender esta relación primero se necesita definir, al menos tentativamente, qué entendemos por ciudad popular y cómo esta definición crea un espacio en común que permita hablar sobre la condición urbana de distintas ciudades de América Latina. Luego se escoge una de estas ciudades y, a partir de ella, se analizará la relación entre los procesos de urbanización y la producción de los espacios públicos. Finalmente, se buscará caracterizar dichos espacios, llegando a conclusiones que nos permitan explorar otras formas de pensar y de planificar nuestras ciudades. 
Es así que este artículo se basa en un estudio de caso desarrollado en el distrito de San Juan de Lurigancho1, en la ciudad de Lima. La investigación se llevó a cabo en la Urbanización Mariscal Cáceres, comparando los procesos de producción de espacios públicos de dos asentamientos: Ciudad de los Constructores (zona plana) y Asociación Familiar Unidos para el Desarrollo (zona de laderas). La primera etapa consistió en caracterizar ambos asentamientos y su consolidación urbana usando una base de datos georreferenciados, proporcionados por el Programa Barrio Mío2 y el Proyecto Urbano Integral Mariscal Cáceres ${ }^{3}$. En la segunda etapa, se realizó un trabajo de campo donde se pudo recorrer y observar los asentamientos, así como llevar a cabo entrevistas a profundidad y conversaciones informales con los residentes. Esto permitió no solo corroborar los datos obtenidos, sino profundizar en la comprensión de los procesos de producción y consolidación del hábitat barrial. Finalmente, este trabajo pretende ser también un aporte al urbanismo latinoamericano desde lo público y no solo desde el problema de la vivienda, dando cuenta de la complejidad propia de nuestra región y de lo urbano como una construcción y experiencia de vida colectiva. Siendo el objetivo principal explorar y analizar la relación entre las diversas formas de urbanización popular y los procesos de producción y apropiación de los espacios públicos.

\section{LA CIUDAD POPULAR: PARADIGMA URBANO LATINOAMERICANO}

La condición urbana de América Latina está directamente relacionada con la condición popular de los procesos de urbanización que han dado forma a la periferia de nuestras ciudades. Es así que las favelas, las villas miseria, las colonias populares, los ranchos, las barriadas o los asentamientos humanos, son fenómenos

1 El distrito de San Juan de Lurigancho se encuentra ubicado al noreste de Lima. Limita por el norte con San Antonio - provincia de Huarochirí-, por el este con San Antonio y Lurigancho-Chosica, por el sur con El Agustino y Lima, y por el oeste con los distritos de Rímac, Independencia, Comas y Carabayllo. La conformación oficial del distrito se dio el 13 de enero de 1967. Los primeros procesos de urbanización conformaron las zonas de Zárate, Caja de Agua o Canto Chico y continuaron su expansión — hacia el este — hasta llegar a la zona conocida como Canto Grande.

2 El Programa Barrio Mío (2012-2014) fue creado mediante la Ordenanza n. ${ }^{\circ}$ 1625, durante la gestión de Susana Villarán (2011-2014). Este programa tenía como objetivo implementar proyectos que prioricen aspectos de desarrollo urbano, prevención del riesgo, cultura, deporte, salud y recreación en zonas vulnerables de Lima. Barrio Mío estaba conformado por dos áreas: Proyectos de Mitigación de Riesgo en Laderas (PMRL) y Proyectos Urbanos Integrales (PUI).

3 Los Proyectos Urbanos Integrales (PUI) fueron instrumentos técnicos-participativos que tenían como objetivo identificar y priorizar una cartera de inversiones urbanas que permitan mejorar la calidad de vida de los barrios. El PUI Mariscal Cáceres (2013) se emplazó en la parte central y oriental del distrito San Juan de Lurigancho - Canto Grande-, comprendiendo un territorio de 4,87 km² aproximadamente. La zona de intervención se ve delimitada por la Av. Wiesse — por el este-, la Av. Héroes del Cenepa o Av. Bayóvar — por el sur-, la Av. Ampliación y la cadena montañosa que divide el distrito de San Juan de Lurigancho de San Antonio de Chaclla en Jicamarca — por el oeste- - El área de trabajo también incluyó parte de la cadena montañosa en su flanco oeste, donde se encuentra la Av. Javier Pérez de Cuéllar. 
comunes y repetitivos en el paisaje urbano. Estos procesos de urbanización popular se han convertido en una constante irreversible $y$, a excepción de países como Argentina, Chile o Uruguay, son la principal característica de nuestra condición urbana latinoamericana (Duhau, 1998).

Los desplazamientos por conflictos armados internos, la aparición de una industria incipiente (1930-1970) en las ciudades — sobre todo en las capitales_, la desarticulación del mundo rural y la concentración de servicios públicos en entornos urbanos, generaron una explosión demográfica cuyo resultado fue la expansión de las manchas urbanas, sustentada en el desarrollo y la producción de urbanizaciones populares al margen de las iniciativas del mercado inmobiliario y las políticas públicas urbanas (Pereyra, 2006). Sin embargo, en muchas ocasiones, estos procesos de ocupación irregular fueron permitidos, asistidos y alentados por los gobiernos que encontraron en aquellos fenómenos no solo una solución de vivienda barata para los sectores más pobres (Connolly, 2013), sino también la posibilidad de establecer redes de clientelismo político útiles para sus propios intereses (Collier, 1978). No obstante, muchas de estas iniciativas de formalización o habilitación urbana coexistieron de forma contradictoria e irregular y carecieron de una voluntad política real para su implementación (Riofrío, 2004; Duhau, 2001). Es decir, la ciudad latinoamericana moderna es el resultado del funcionamiento y la coordinación de dos lógicas: la del mercado y la del Estado, pero también de una tercera: la lógica de la necesidad (Abramo, 2012). Esta necesidad, encarnada en las personas que habitan tales asentamientos, ha consistido en habilitar progresivamente el espacio conquistado, de tal manera que lo que originalmente era cerro, arenal, tierras eriazas, bosques o zonas residuales se convierta en parte de la ciudad ante la falta de habilitaciones urbanas por parte del Estado o del mercado formal (Riofrío \& Driant, 1987). Estas ocupaciones son desarrolladas por personas organizadas de forma comunal o familiar a través del mercado informal de suelo, por traficantes de suelo que inician procesos de lotización ilegales con fines de lucro, constituyendo mafias organizadas y delictivas, y por gobiernos municipales cuyas iniciativas promueven estas urbanizaciones ilegales como parte de las redes de corrupción (Calderón Cockburn, 2016).

Ahora, si bien el hábitat popular también puede referirse a cualquier tipo de edificación que habiten las clases trabajadoras más pobres, como tugurios alquilados en el centro de las ciudades o viviendas de interés social (Connolly, 2013; Jaramillo, 2013), esta investigación se enfoca en desarrollar este concepto centrándose en asentamientos humanos autoproducidos y consolidados de forma irregular e ilegal en la periferia.

Asimismo, la ciudad popular, como categoría de análisis, permite trascender la dualidad entre lo formal y lo informal. Primero, porque la informalidad no puede resumirse a un conflicto con el Estado o el mercado formal o a un incumplimiento de sus normativas dado que, generalmente, las actividades de producción informal del espacio se retroalimentan o vinculan —en diversos grados y formas - con los gobiernos locales, las políticas públicas o los mercados denominados «formales» (Herzer et al., 2008; Roy, 2005). En segundo lugar, la irregularidad o informalidad 
urbana no es una característica que se despliega de manera homogénea sobre el territorio, sino que puede ser desagregada en tres formas: urbanística, constructiva y jurídica (Abramo, 2012), superponiéndose y produciendo diversos tipos de tejido urbano.

Es así que el despliegue de múltiples formas de producción del hábitat urbano, que oscilan continuamente entre la irregularidad y la regularidad, conforman la estructura socioespacial de la ciudad popular. Para caracterizar este proceso, centrándose en la relación entre el mercado de suelo y el acceso a la vivienda, P. Abramo (2012) propone el concepto de ciudad com-fusa donde, según los tipos de mercado de suelo, se puede evidenciar el surgimiento de una ciudad popular compacta caracterizada por procesos de consolidación y densificación; y una ciudad popular difusa que se expande a través de procesos informales de lotización. Es decir, la ciudad popular produce distintas formas de hábitat, que la hacen heterogénea y diversa, sin perder una serie de características y problemáticas comunes. Todo esto hace necesario generar estudios y metodologías para analizar, no solo la vivienda (Riofrío, 2004; Calderón \& Maquet, 1990), sino también las formas en que se produce lo público dentro de los procesos de urbanización y consolidación del hábitat popular.

Actualmente, la periferia de las manchas urbanas latinoamericanas crece más por lógicas de especulación y tráfico de suelo que por una presión demográfica venida de migraciones rurales (Carrión, 2016). Asimismo, los procesos de urbanización irregulares e ilegales que producen la ciudad popular son la sumatoria de fuerzas estructurantes de los mercados, de la agencia del Estado como coordinador de recursos públicos y planificación institucional, y de la necesidad de determinados sectores de la población por autoproducir sus barrios, viviendas y espacios públicos. Esto hace de la ciudad popular un tejido urbano heterogéneo, donde existen distintos intereses y esfuerzos de producción del hábitat y, por lo tanto, de sus espacios públicos. Esto permite plantearnos una segunda pregunta: ¿Qué tipos de espacios públicos se producen a partir de los diferentes procesos de urbanización popular?

\section{LIMA: LA CIUDAD DE LAS LADERAS 4}

En el Perú uno de los acontecimientos más importantes ocurridos en el siglo XX fue el proceso migratorio de poblaciones del mundo rural a entornos urbanos ubicados, sobre todo, en la costa. En el caso de Lima, estos desplazamientos de población se tradujeron en masivos y acelerados procesos de urbanización que se desarrollaron, en una primera etapa, sobre terrenos marginales en zona plana o semiplana, ubicados fuera del casco urbano tradicional de la ciudad. Desde finales

\footnotetext{
4 Subtítulo basado en el libro: La Ciudad de las Laderas: Workshop Limápolis 2016 (Rodríguez \& Muñoz, eds., 2017a). Trabajo que presenta (1) los resultados del taller de proyectos realizado en torno al «Workshop Internacional Limápolis 2016», organizado por la Facultad de Arquitectura y Urbanismo de la Pontificia Universidad Católica del Perú; así como (2) diversos ensayos de reflexión urbanística y arquitectónica.
} 
del siglo XX e inicios del siglo XXI, estos procesos de urbanización se dieron en una segunda etapa, sobre terrenos residuales y en zona de laderas5, que presentan condiciones topográficas nuevas para la autoproducción del entorno urbano y la consolidación del hábitat (Velarde \& Vásquez, 2017).

Es así que actualmente Lima Metropolitana6, en el caso de algunos distritos periféricos, está compuesta por una ciudad popular consolidada y en proceso de densificación, ubicada en las zonas planas. Asimismo, está compuesta por otras nuevas - y no tan nuevas - ocupaciones populares, que se encuentran en simultánea consolidación y expansión difusa, localizadas en zonas de pendiente o laderas, y que representan el 15\% de la mancha urbana (Rodríguez, 2016)7.

Es decir, no existe un modelo integral de ciudad en laderas para las clases populares en Lima propuesto por el Estado o el mercado formal. A esta ausencia se le suma el mercado informal de suelo y el tráfico de terrenos, como principales medios para acceder al lote urbano. Lo cual genera: (1) que en los asentamientos que surgen del mercado informal, debido a su lógica lucrativa y especulativa en beneficio de agentes privados, lo público resulte un concepto y espacio marginal; (2) que las mafias de traficantes de suelo invadan u ocupen los pocos espacios reservados para áreas comunes o bordes verdes, de asentamientos humanos anteriormente establecidos; y (3) que algunas veces los dirigentes vecinales acepten comercializar para beneficio propio las pocas áreas reservadas para espacios públicos, formando «ampliaciones» sobre los asentamientos iniciales (Velarde Herz, 2017).

Asimismo, una de las mayores dificultades de los asentamientos ubicados en zona de laderas es poder vincular la movilidad con la vivienda y el espacio público (Kahatt, 2017). Esto se debe a los retos urbanísticos que ofrecen los cambios en la pendiente, pero, sobre todo, a la falta de una proyección y consolidación integral de los componentes urbanos como partes de un sistema planificado. Por lo tanto, pensar nuestras laderas es asumir su heterogeneidad geográfica y la de los tejidos urbanos que ahí se producen, así como comprender las lógicas del mercado informal de suelo, la planificación comunitaria del hábitat y el rol que desempeña la política y la inversión públicas.

5 En la ciudad de Lima, la mayoría de los terrenos planos y semiplanos fueron ocupados hasta el año 1985, llegando hasta pendientes de $20 \%$ de inclinación (primera línea de quiebre). A finales de la década de 1980, comienzan a ocuparse las zonas de ladera con pendientes de hasta 35\% o incluso más (segunda línea de quiebre). El límite habitable planteado para la zona de ladera es de 35\%, por constituir zonas de riesgo y presentar dificultades para el abastecimiento de servicios básicos y consolidación física de los barrios (Muñoz \& Rodríguez, 2015).

6 El área metropolitana de Lima comprende 49 distritos que son parte de la Provincia de Lima y la Provincia Constitucional del Callao. La población total del área metropolitana es de 9752000 habitantes, siendo Lima Este la zona más poblada de la ciudad con 2619814 pobladores (Instituto Nacional de Estadística e Informática [INEI], 2014).

7 Si bien un 15\% de la mancha urbana de Lima Metropolitana se encuentra en zona de pendiente, en su totalidad, las laderas de Lima - habitadas y sin habitar- ocupan un $70 \%$ de su territorio (Ludeña, 2016); por lo que representan un importante espacio de inevitable expansión —informal o formal- de nuestra ciudad, que necesita ser estudiado, pensado urbanísticamente y planificado de manera integral. 


\section{CIUDADES POPULARES: CIUDADELA MARISCAL CÁCERES}

En el distrito de San Juan de Lurigancho, en la década de 1970, se desarrolló un proceso de urbanización popular masivo; como consecuencia de ello, se planteó desarrollar una ciudad satélite. Esta iniciativa se postergó hasta la década de 1980, cuando se planificó la «Ciudad Satélite Bolognesi» como un programa de vivienda para la clase trabajadora. El proyecto fue pensado en cuatro etapas, las cuales incluyeron módulos de vivienda, accesos, servicios básicos y espacios públicos. La obra fue implementada en 1986 pero, debido a la crisis económica por la que atravesaba el Perú, se lotizó y se sortearon las propiedades a través de ENACE8, sin mayor tipo de habilitación urbana y cambiando el nombre del proyecto a «Ciudadela Mariscal Cáceres» (Mejía Carrión, 2009).

Una vez adquiridos los lotes —etapas I y II—, los propietarios fueron ocupando la zona y construyendo sus viviendas de manera progresiva. Este fue el primer y único proceso de urbanización planificado por el Estado como parte del proyecto. El suelo destinado a las otras dos etapas — III y IV—, donde se acumuló basura o restos de material de construcción de los procesos de edificación de los barrios, empezó a ser ocupado informalmente por los obreros que trabajaron en la construcción de algunas viviendas de las etapas I y II. Es así que a inicios de 1990, estas nuevas ocupaciones informales recibieron la ayuda de ENACE y FONAVI9, para la planificación de sus barrios, la formalización de sus lotes y el acceso a créditos para la edificación de sus viviendas, integrándose al proyecto urbano.

En la zona plana también existió la planificación de espacios públicos que denominaremos «espacios públicos convencionales» (fig. 1). Es decir, espacios públicos delimitados e integrados a la trama y al sistema urbano; con una distribución de usos y espacios claros (fig. 2); compuestos por mobiliario o equipamiento público, tanto al aire libre como cerrado; y cuya producción y gestión obedece a la planificación pública. Esta red de espacios se vio conformada por largas alamedas que funcionarían como espacios recreativos, corredores peatonales y áreas verdes, así como áreas reservadas para parques o equipamiento educativo. Esto se debe a que, a lo largo del siglo XX, existieron

8 Empresa Nacional de Edificaciones (ENACE), fundada en 1981 sobre lo que fue la Empresa de Administración de Inmuebles del Perú (EMADI-PERÚ). El 7 de mayo de 1992, pasó a formar parte del sector institucional del Ministerio de la Presidencia. Actualmente, su capital social pertenece al Fondo Nacional de Financiamiento de la Actividad Empresarial del Estado (FONAFE), integrando el Sector de Economía y Finanzas dentro del Estado peruano.

9 El Fondo Nacional de Vivienda (FONAVI) fue creado en 1979 por el Gobierno Revolucionario de las Fuerzas Armadas, en su segunda etapa, con Francisco Morales Bermúdez como presidente. EI FONAVI se basaba en una contribución obligatoria de los trabajadores que buscaba financiar, en un futuro, la construcción o adquisición de viviendas propias. Este fondo duró hasta 1998, cuando le cambiaron de nombre a Impuesto Extraordinario de Solidaridad. En 2004, se derogó tras irregularidades en sus cifras. 


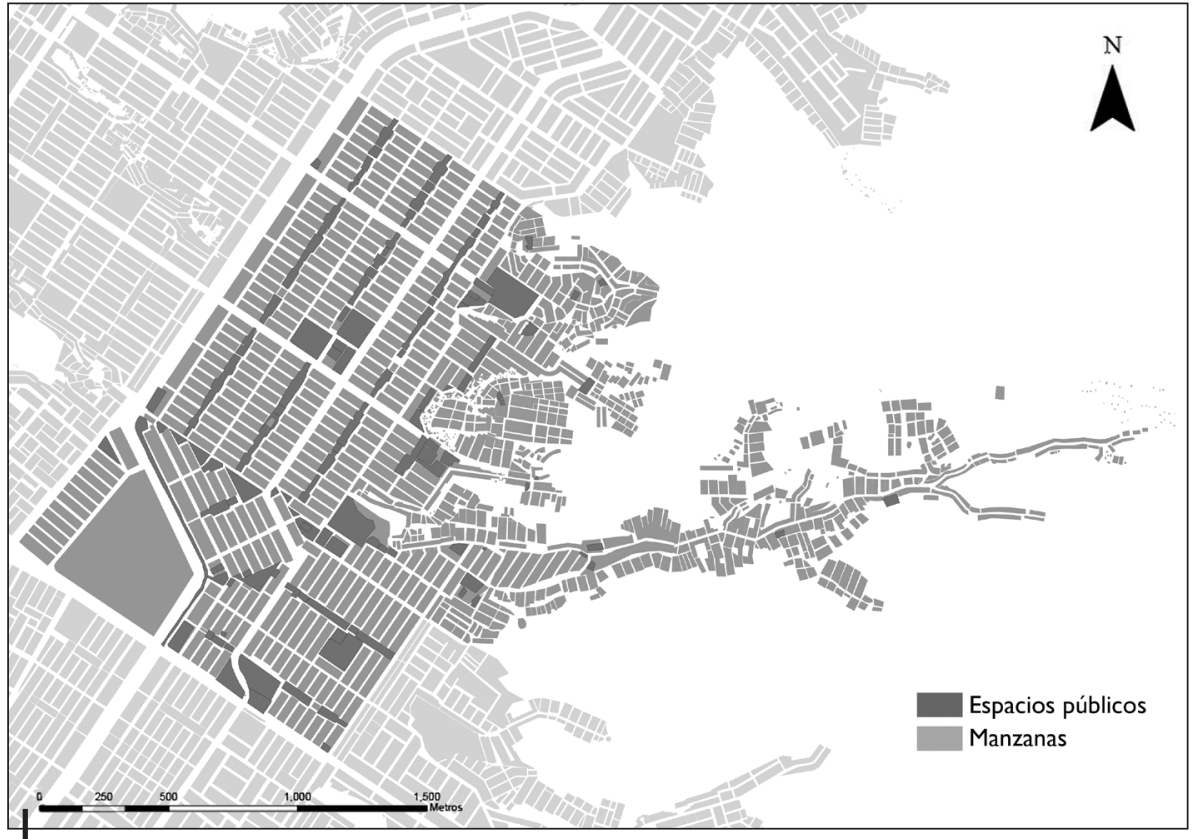

Figura 1 - Distribución de espacios y servicios públicos en Mariscal Cáceres

Fuente: Elaboración propia, basado en el PUI Mariscal Cáceres

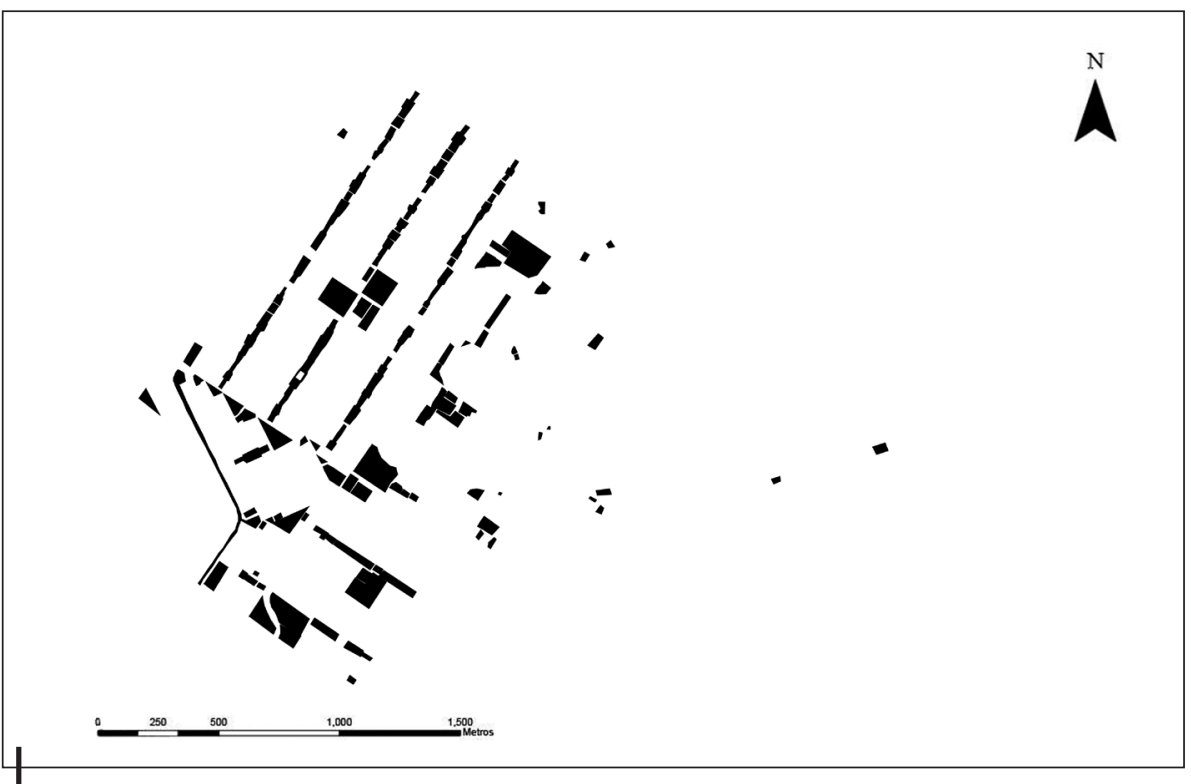

Figura 2 - Esquema de distribución de espacios y servicios públicos en Mariscal Cáceres Fuente: Elaboración propia, basado en el PUI Mariscal Cáceres 
instituciones estatales que buscaron ofrecer asistencia urbanística y/o constructiva a los barrios populares 10 .

Aunque muchas de estas iniciativas no pudieron ser implementadas con la misma velocidad con la que se desarrollaban los procesos de urbanización irregular y no siempre han asegurado la consolidación integral de los asentamientos, significaron un esfuerzo por ordenar la ciudad popular desde el Estado y la población organizada.

Sin embargo, en estos asentamientos, herederos de una planificación estatal débil pero oportuna, la consolidación —no siempre exitosa- de los espacios públicos convencionales se ha desarrollado a través de largos y variados procesos de autoproducción y autogestión colectiva desplegados por las juntas vecinales o algunas familias. Esto hace que existan formas y estrategias flexibles de apropiación o uso, que varían a través del tiempo según las necesidades de la comunidad11. Es justamente esta dinámica intensa, la confianza en el mundo de la calle y la capacidad de las personas de transformar, apropiarse y habilitar colectivamente un espacio a través de actividades autogestionarias, lo que posibilita que estos espacios aparentemente vacíos, sin límites definidos, inacabados, expectantes y sin equipamiento o mobiliario, cobren vida pública y se integren al sistema urbano (Takano \& Tokeshi, 2007; Hernández Bonilla, 2008). De igual manera, de existir algún tipo de asistencia del gobierno municipal, esta se desarrolla de forma fragmentada, limitada y poco participativa, lo cual no permitió asegurar el adecuado mantenimiento de los espacios a través del tiempo.

Estos espacios públicos convencionales, similares a los que se pueden encontrar en cualquier otra parte de la ciudad — parques, losas deportivas o alamedas-, pero donde los procesos de producción del espacio involucran formas de planificación comunal, las asambleas barriales, la mano de obra y el dinero invertido por parte de la comunidad, son denominados «espacios públicos populares convencionales». Es decir, son espacios relativamente delimitados e integrados a la trama urbana, en simultáneo proceso de consolidación y deterioro, con formas de apropiación y usos flexibles a lo largo del tiempo y según las necesidades de la comunidad. Dichos espacios pueden estar compuestos o no por algún tipo de mobiliario permanente o momentáneo, y su producción y gestión es comunitaria o familiar, y a veces asistida o compartida con el gobierno municipal.

10 En 1961 se promulgó la Ley 13517, también llamada «Ley de Remodelación, Saneamiento y Legalización de los barrios marginales». Era una iniciativa de planificación urbana y de apoyo a las urbanizaciones irregulares pionera en América Latina. Esta ley prohibirá la formación de barriadas por medio de invasiones después de setiembre de 1960, encargándole la formación y consolidación de barriadas al Estado que, a través de distintas instituciones, debía trazar las calles, proyectar los espacios públicos, instalar servicios — luz, agua y desagüe_ - y administrar la cantidad y el tamaño de los lotes de vivienda. Una vez terminada esta habilitación urbana, la población podía recién ocupar el suelo y empezar la construcción de sus viviendas y de su entorno urbano.

11 Un espacio público popular convencional puede variar en usos, mobiliario y equipamiento según las necesidades de la comunidad circundante. Es decir, puede ser un mercado, un jardín de niños, un área verde o una loza deportiva durante un determinado tiempo, y según las necesidades que la comunidad presente en momentos particulares. 
De esta manera, la asistencia y la planificación urbanística por parte del Estado, al inicio del asentamiento, permitieron que la red de espacios públicos sea visible y forme parte del sistema urbano, originando que, si bien estos espacios usualmente se encuentran en constante consolidación y deterioro afectando la variedad e intensidad de usos, formen parte activa de la vida en el barrio.

\section{LA VIDA ENTRE LADERAS}

En la década de 1990, se aplicaron medidas de reajuste económico que desarticularon las instituciones que buscaban planificar y ordenar las ciudades en el Perú12. Es decir, se redujeron las regulaciones sobre los territorios y la acción estatal pasó a centrarse en la entrega de títulos de propiedad y el saneamiento de servicios básicos (Calderón Cockburn, 2016). Este abandono del Estado, el cual dejó de pensar la ciudad de forma integral, instituyó la propiedad privada como unidad básica de gestión de las ciudades y coincidió con la ocupación masiva de las zonas de ladera de Lima. Es así que, a inicios de 1990, cuando los procesos de urbanización desbordaron la trama urbana inicial de Mariscal Cáceres y sus ampliaciones asistidas por el Estado, no existió un esfuerzo de planificación urbana integral.

Por otro lado, debido a la geografía accidentada, en la zona de laderas los habitantes no pudieron replicar los mismos patrones, estrategias y experiencias de urbanización de sus antecesores ubicados en la zona plana, desarticulándose la trama urbana conforme se la iba ocupando (fig. 3). Este proceso no se dio de forma abrupta, sino que generó una zona de tránsito denominada «línea de quiebre» por el equipo del PUI Mariscal Cáceres (fig. 4). Esta área supone una ruptura geográfica, urbanística y de las formas de habitar entre la zona plana y semiplana — ciudad compacta — y la zona de laderas — ciudad difusa — (Rodríguez \& Muñoz, 2017b). Es así que, a diferencia de la zona plana, donde existió la asistencia —no siempre exitosa - de instituciones o empresas públicas encargadas de planificar y ayudar a consolidar los barrios, en la zona de laderas no se pensó —ni se ha pensado aún a nivel estatal — un modelo de ciudad. De esta manera, las formas de planificación y producción del espacio que se despliegan en la zona de laderas, suelen darse a partir de un esfuerzo de planificación comunitario (fig. 5), por parte de poblaciones que buscan pensar su asentamiento o de urbanizadores informales quienes lotizan y disponen de la distribución y localización de los componentes en la trama urbana, así como de los usos del suelo.

Por lo tanto, se trata de un área en la que no existe una planificación pública o comunitaria integral del hábitat popular, debido a que: (1) no se pudieron

12 Durante el Gobierno de Alberto Fujimori (1990-2000) se aprobó la Constitución de 1993 que eliminó el derecho a la vivienda. También se desarticuló el Ministerio de Vivienda y Construcción, el Banco de la Vivienda y el Banco Central Hipotecario. Asimismo, en 1995 se aprobó la Ley de Tierras —Ley 26506 - , eliminando la intangibilidad del suelo agrícola y anulando así la política de límites urbanos, que había sido precariamente implementada hasta ese momento (Calderón Cockburn, 2016). 


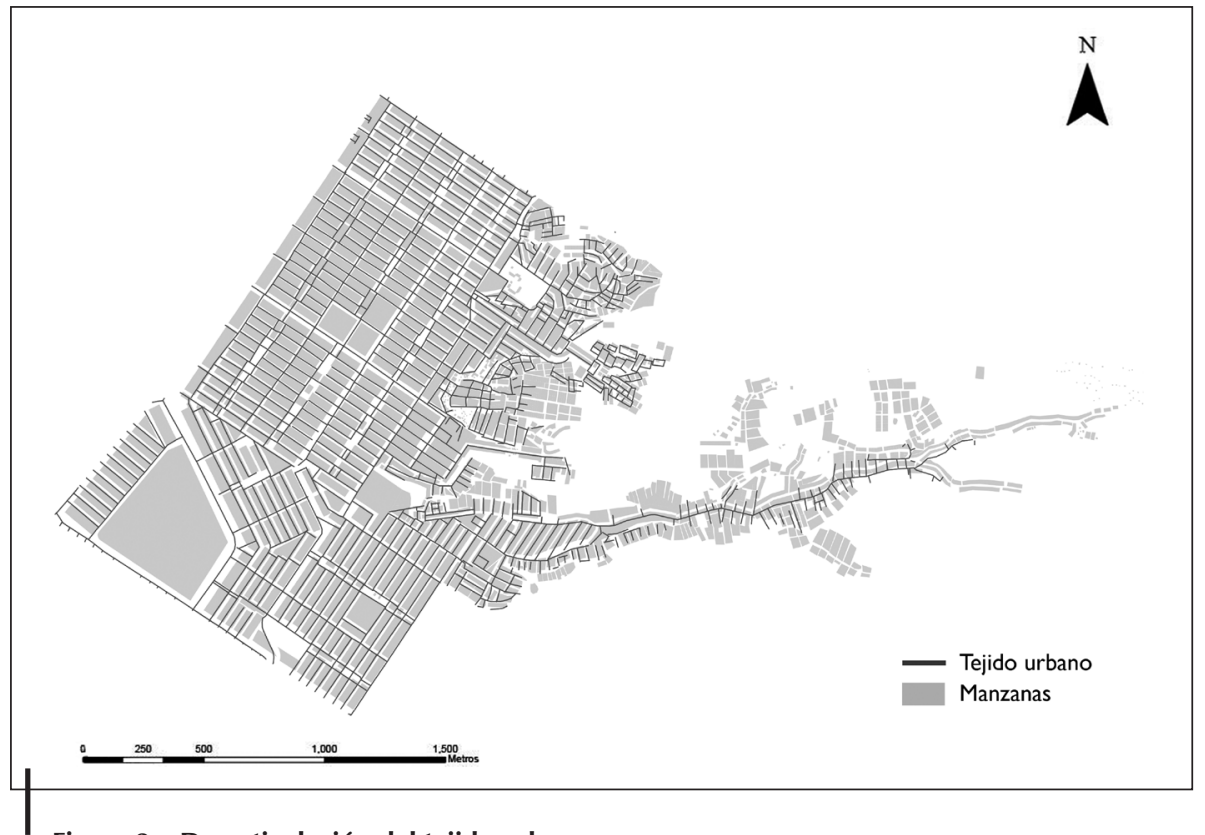

Figura 3 - Desarticulación del tejido urbano

Fuente: Elaboración propia, basado en el PUI Mariscal Cáceres

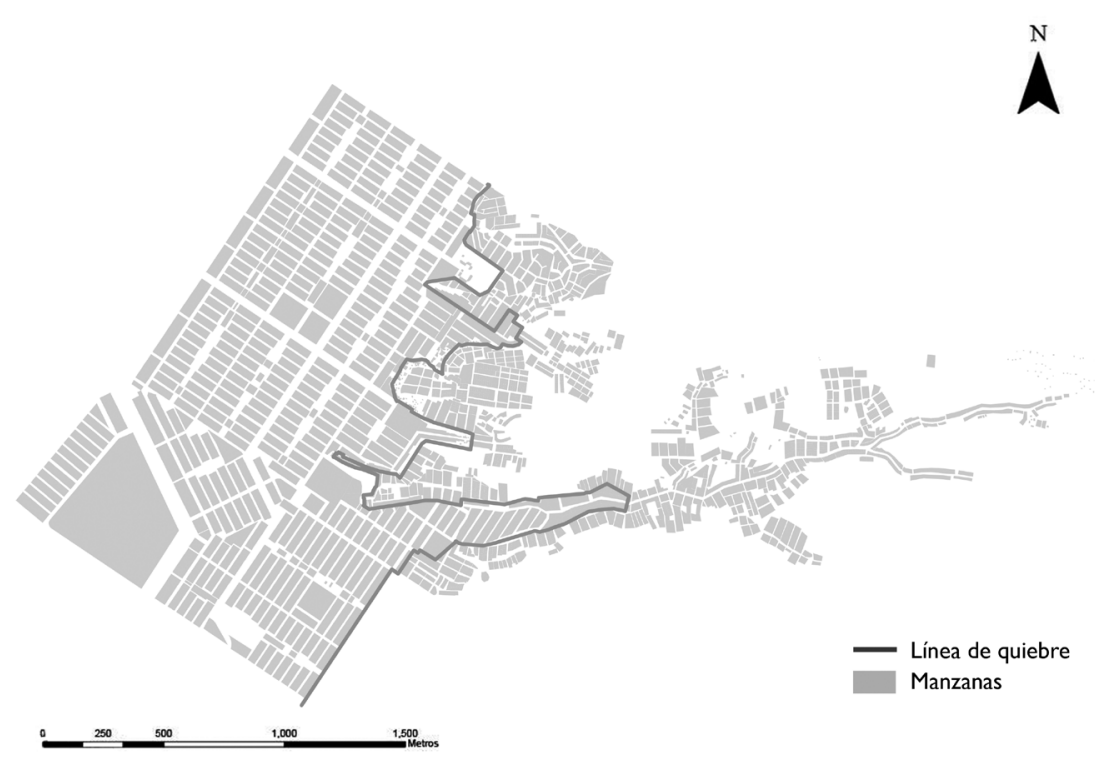

Figura 4 - Línea de quiebre propuesta por el PUI Mariscal Cáceres

Fuente: Elaboración propia, basado en el PUI Mariscal Cáceres 


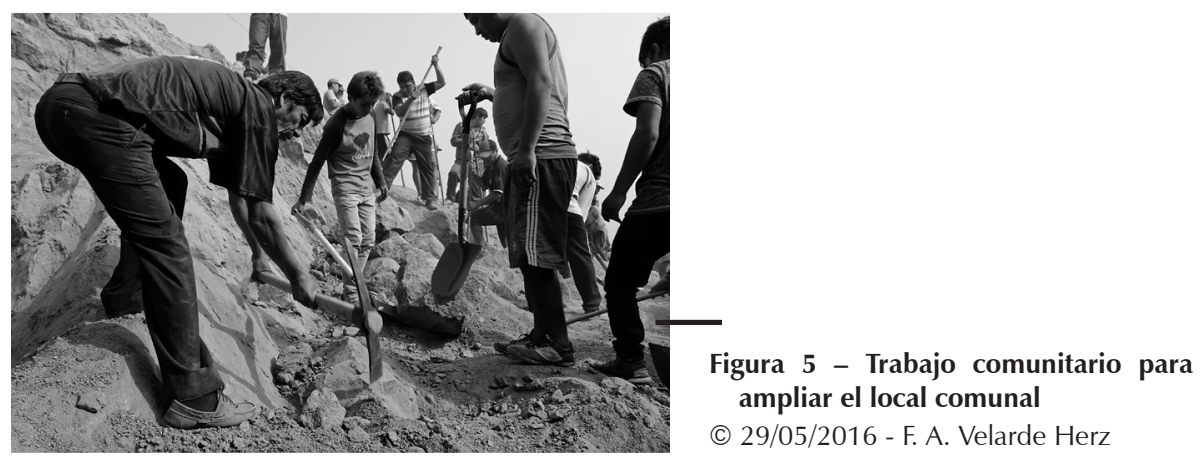

reproducir las experiencias previas de ocupación y consolidación desarrolladas en la zona plana; (2) la ladera se encuentra fragmentada políticamente en múltiples asentamientos humanos que no permiten una planificación integral del territorio desde las juntas vecinales; (3) no existen referentes o experiencias públicas por generar una traducción espacial de los componentes urbanos de la zona plana a la zona de laderas; (4) la pendiente aparece como un nuevo escenario geográfico y reto urbano donde desarrollar la producción de un hábitat popular de calidad.

De esta forma, en la zona de laderas, el lote se constituye como la unidad básica de expansión y proyección difusa del tejido físico. Asimismo, los accesos - escaleras y caminos-, usualmente con orientación vertical, son otra prioridad dentro de los usos del suelo, dado que a través de ellos se ingresa materiales de construcción para los procesos de edificación. Estos accesos son por mucho tiempo los únicos espacios de encuentro que tienen los vecinos y vecinas de estos asentamientos, convirtiéndose muchas veces en los primeros espacios de vida colectiva.

Es así que, en la zona de laderas, usualmente el espacio público se encuentra entre los últimos componentes urbanos a pensar o consolidar puesto que: (1) la construcción de las viviendas ha ocupado gran parte del espacio disponible en el asentamiento; (2) la pendiente dificulta muchas veces la réplica de espacios públicos convencionales; (3) en muchos asentamientos la consolidación del hábitat es precaria, lenta y ausente de inversión pública, debido a la irregularidad en la propiedad del suelo13; y finalmente, (4) producto del tráfico de terrenos se han invadido los pocas áreas reservadas para espacios comunes o ampliaciones. Sin embargo, como se menciona anteriormente, esto no impide que exista una

13 La Asociación Unidos al Desarrollo se formó en 2001. En el año 2007, la municipalidad de San Juan de Lurigancho les otorgó la constancia de posesión, con lo que pudieron tramitar algunos servicios básicos más adelante. Por ejemplo, la luz eléctrica recién fue instalada en 2010. Al no existir aún accesos, la empresa de luz dejó los postes de concreto en la Av. Central —ubicada en la zona plana- y fueron las vecinas y vecinos quienes tuvieron que cargarlos y transportarlos hasta su asentamiento, poste por poste. Hasta la actualidad, no cuentan con título de propiedad. Otros asentamientos en zona de laderas, sin título de propiedad o constancia de posesión municipal, realizan instalaciones informales de algunos servicios. 
dimensión colectiva y que esta no se manifieste como componente urbano dentro de la trama física.

Los encuentros casuales, actividades opcionales o sociales (Gehl, 2013), en este caso, suelen darse en las escaleras y los retiros - patios - de las viviendas que se encuentran abiertos y expuestos. Aunque muchos de estos espacios podrían ser cuestionados como áreas públicas en un sentido convencional, son las difusas líneas entre lo público y lo privado, y las intensas formas de apropiación familiar, lo que convierte a estos espacios en «espacios semicomunales». Se les denomina de esta manera dado que son los vínculos comunitarios de cercanía y empatía los que permiten, sostienen y potencian la interacción entre conocidos ${ }^{14}$. Es decir, más que lo desconocido o la alteridad — tolerar y respetar al otro- como punto de partida para construir lo público (Carrión, 2007), es lo conocido y lo común lo que posibilita el poder coordinar acciones comunitarias y sostener encuentros casuales en espacios semicomunitarios.

En los retiros o terrazas ubicados frente a las viviendas, y que usualmente se encuentran cercanas a las escaleras, la gente trabaja, siembra jardines, juega, se encuentra y conversa; pero, sobre todo, son espacios donde están dispuestos a ser observados en sus distintas actividades cotidianas y, al mismo tiempo, buscan poder observar lo que sucede a su alrededor. Si bien por momentos pueden albergar usos colectivos, no dejan de ser espacios privados, ya que forman parte de las viviendas. En otras palabras, si una persona decide que ya han jugado demasiado tiempo los niños y niñas por su patio, tiene la posibilidad de pedirles que se retiren, lo que evidencia la característica principal de los espacios semicomunales: la capacidad de atraer y sostener actividades colectivas sin perder su cualidad de espacio privado, lo que permite al propietario controlar dichas actividades colectivas, evitarlas o prohibirlas cuando lo vea conveniente (Velarde Herz, 2017).

De igual forma, cada familia considera «su escalera» a las gradas que se encuentran frente a su vivienda y, por lo tanto, dispone de ella. Al ser espacios de tránsito, es recurrente encontrarse con conocidos y sostener pequeñas conversaciones, convirtiendo las escaleras en espacios semicomunales alargados que trepan la ladera formando líneas y que intensifican su diversidad e intensidad de usos, según su grado de consolidación (figs. 6 y 7) y el número de viviendas con retiros que se encuentren en ellas. Por otro lado, una escalera en malas condiciones y sin retiros suele ser un espacio únicamente de tránsito y sin posibilidad de generar mayor interacción.

14 En la Asociación Familiar Unidos al Desarrollo, para que una persona pudiera ingresar a la asociación y ocupar un lote se le pedía antecedentes policiales y se decidía su admisión en una asamblea. Usualmente, también se recomendaba invitar a familiares o conocidos, por lo que el control de quienes integraban el asentamiento es riguroso. Esto, sumado a la pendiente, que permite observar y controlar los pocos accesos al asentamiento, genera una comunidad cerrada. Estas prácticas y características suelen repetirse en los demás asentamientos de la zona de laderas. A diferencia de esto, en la zona plana, actualmente, los vecinos originales se han mudado y existe un mercado de alquiler que permite la rotación de población. Sin embargo, al existir todavía lógicas barriales, muchas personas se conocen y reconocen en las calles, aunque con menos intensidad que en la zona de laderas. Es decir, en la zona plana coexisten los desconocidos con los conocidos. 


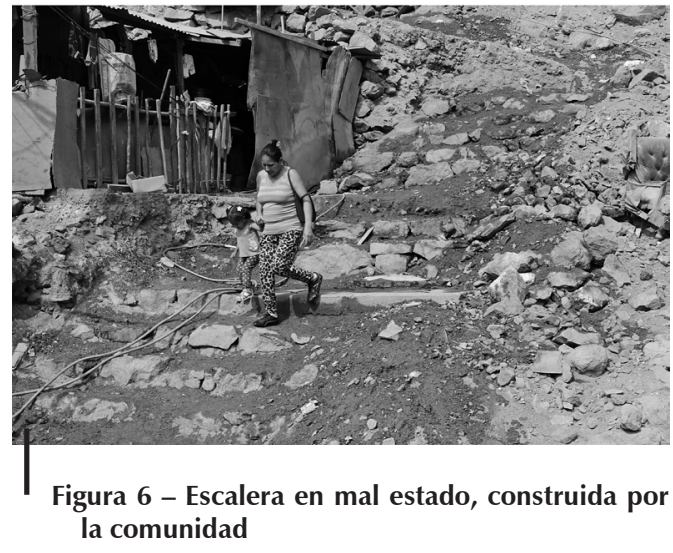

(C) 29/05/2016 - F. A. Velarde Herz

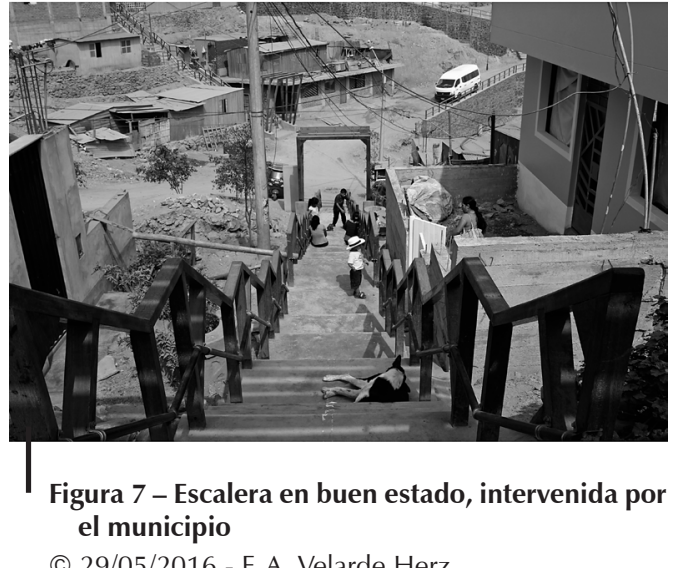

(C) 29/05/2016 - F. A. Velarde Herz

Las actividades que se pueden observar en estos espacios son desplegadas por tiempos relativamente prolongados y sin existir un límite claro entre la escalera, los retiros y las viviendas (fig. 8). Es difícil establecer una jerarquía entre los espacios debido a lo permeable de la trama del asentamiento, los bordes difusos, los materiales de construcción de las viviendas y la inclinación de la pendiente. Esto último, por ejemplo, posibilita conectar el techo de una vivienda - a veces solo usando una tabla de madera - con una escalera u otro espacio comunal ubicado en la parte superior, convirtiendo al techo de la vivienda en una extensión provisional e improvisada de un espacio semicomunitario para alguna actividad en concreto y por un corto lapso de tiempo.

En estos espacios semicomunales se puede encontrar durante el día, la tarde y con menor recurrencia en las noches a jóvenes jugando (fig. 9), adultos conversando (fig. 10), niños leyendo (fig. 11) o simplemente personas sentadas observando lo que sucede a su alrededor o el paisaje urbano que ofrece la ladera. A saber, son actividades que podrían encontrarse en un espacio público barrial convencional de la ciudad, aunque dada la disposición difusa de estos espacios semicomunales, estas actividades se extienden de forma dispersa por todo el asentamiento. Asimismo, debido a la pendiente, están a la vista de la mayoría de sus habitantes, lo cual aumenta la sensación de simultaneidad y superposición de actividades. Esto último también permite a las familias mantener un control sobre el juego de los niños o niñas, y a la comunidad sobre su asentamiento (fig. 12).

Sin embargo, esta red dispersa de pequeños espacios semicomunales dentro de un asentamiento no es producida de forma planificada o premeditada por cada familia. Muchas veces, estos retiros o terrazas se producen debido a que las viviendas no se encuentran totalmente consolidadas y aún no ocupan el total de la plataforma o el terreno que les corresponde. Probablemente, cuando la vivienda termine de ser construida por sus propietarios, estos espacios desaparezcan y quede hacia la escalera solamente un muro ciego o una puerta, lo cual —como se explicó anteriormente- disminuiría la dinámica comunal de encuentros 


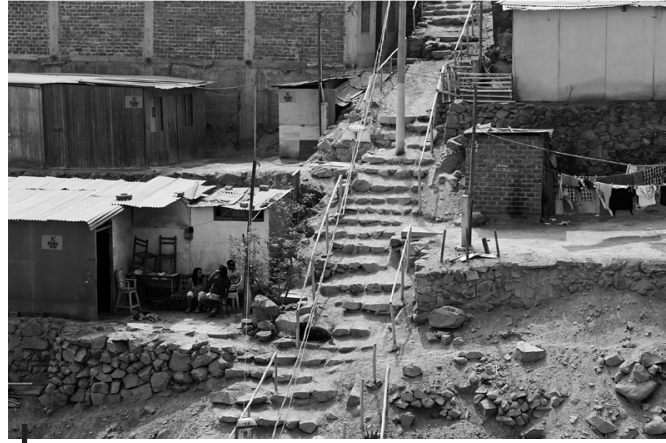

Figura 8 - Retiros de las viviendas como espacios semipúblicos

(c) 29/05/2016 - F. A. Velarde Herz

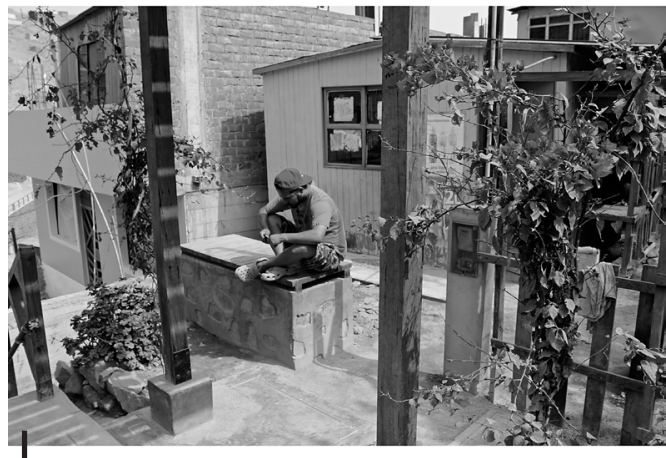

Figura 10 - Persona conversando por celular en el retiro de su casa

(c) 29/05/2016 - F. A. Velarde Herz

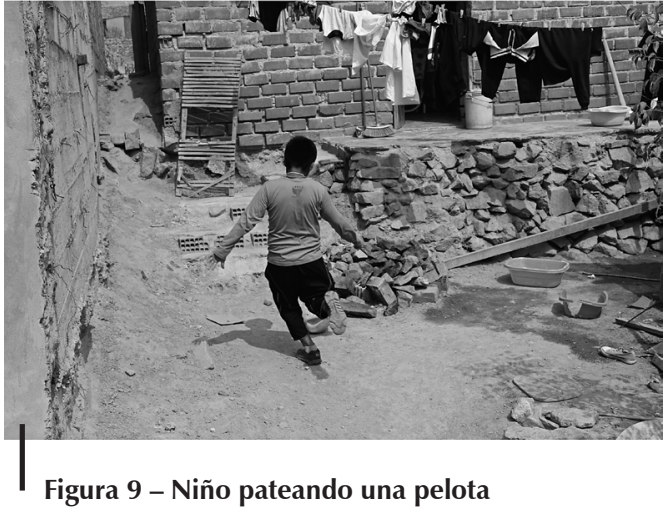

(C) 29/05/2016 - F. A. Velarde Herz

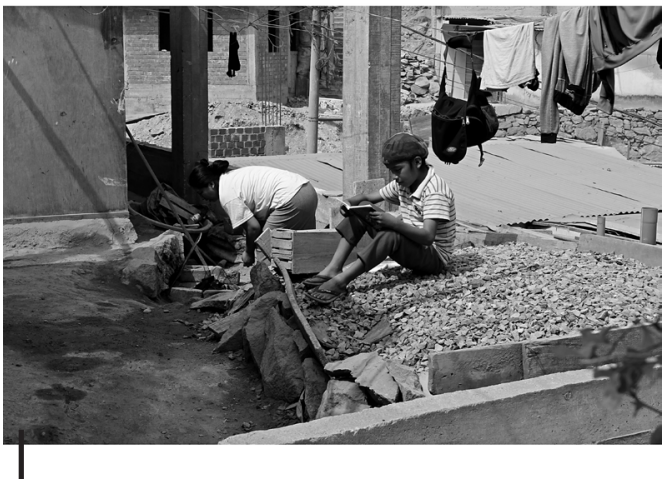

Figura 11 - Niño leyendo en el retiro de su casa

(C) 29/05/2016 - F. A. Velarde Herz

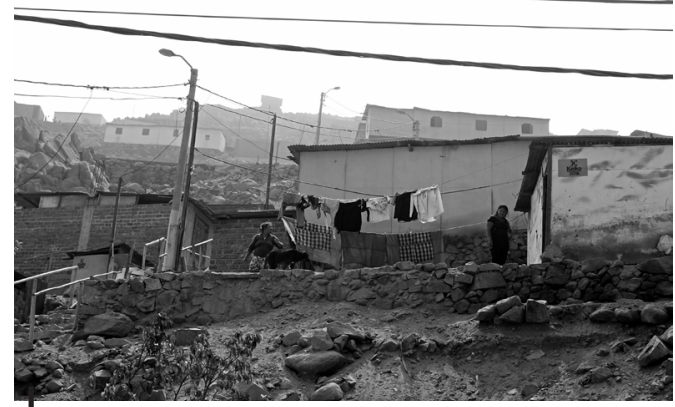

Figura 12 - Mujeres observando el asentamiento mientras realizan labores de cuidado

(C) 29/05/2016 - F. A. Velarde Herz 
y actividades en dicho espacio. No obstante, existen vecinas y vecinos que sí buscan conservar y mejorar estas terrazas, por lo que la evolución espacial de un asentamiento puede conservar algunos espacios semicomunales. Pese a esto, al no existir una planificación conjunta e integral de la ladera, que busque potenciar y producir espacios de encuentro y vida comunal, esta decisión recaerá sobre y variará según la disposición de cada familia o propietario como parte de los procesos de consolidación de su vivienda y no como parte de una planificación integral del asentamiento.

\section{CONCLUSIONES}

En las laderas, los espacios semicomunales surgen por la precariedad y la ausencia de una vivienda construida que aproveche la totalidad del lote o la plataforma. Estos espacios se entrelazan a los caminos y las escaleras conformando una red dispersa y difusa a lo largo de los asentamientos. Su distribución, el carácter expuesto y la inclinación de la pendiente promueven una participación activa y pasiva de los vecinos y vecinas, permitiéndoles observar, escuchar, encontrarse con otras personas e interactuar diariamente. Por ello, los espacios semicomunales no son espacios de transición hacia el espacio público convencional, sino que son la adaptación espacial de un componente urbano —el espacio públicoa un nuevo contexto geográfico: la ladera, donde se desarrollan procesos de urbanización irregulares y difusos, que producen una trama urbana fragmentada. Sin embargo, la existencia de una red de espacios semicomunales no ha significado que los vecinos y vecinas no necesiten o deseen producir espacios públicos convencionales. En el asentamiento «Unidos al Desarrollo», existe un interés por construir lozas deportivas o generar áreas verdes en la ladera. Pero, a diferencia de la zona plana en sus inicios, esta población no cuenta con la asistencia técnica —ni financiera- del Estado para lograr sus objetivos de una forma integral, debido a que las laderas de Lima son un área en la que no existe una planificación pública que acompañe o asista los procesos de urbanización o consolidación del hábitat popular.

Por lo que, de existir una planificación institucional de la ciudad en ladera, podrían tomarse en cuenta los retiros de las viviendas, hoy pasibles de ser regulados por las municipalidades distritales de acuerdo a la normativa vigente. De allí que los gobiernos locales podrían, mediante regulaciones urbanísticas, aprovechar su potencial para convertirlos en espacios para el encuentro y la convivencia, en el marco de propuestas urbanísticas y arquitectónicas integrales. 
El espacio público en la ciudad popular: la vida entre laderas

\section{Referencias citadas}

ABRAMO, P., 2012 - La ciudad com-fusa: mercado y producción de la estructura urbana en las grandes metrópolis latinoamericanas. EURE, 38 (114): 35-69.

CALDERÓN COCKBURN, J., 2016 - La ciudad ilegal. Lima en el siglo XX, 398 pp.; Lima: Punto Cardinal.

CALDERÓN, J. \& MAQUET, P., 1990 - Las ideas urbanas en el Perú (1958-1989), 172 pp.; Lima: Instituto de Desarrollo Urbano (CENCA).

CARRIÓN, F., 2007 - Espacio público: punto de partida para la alteridad. Disponible en http://www.flacso.org.ec/docs/artfcalteridad.pdf (consulta realizada el 20 de octubre de 2016).

CARRIÓN, F., 2016 - Nuevos patrones de urbanización, nueva ciudad en América Latina. In: Ciudades para cambiar la vida. Una respuesta a Hábitat III (J. Borja, F. Carrión \& M. Corti, eds.): 290-310; Buenos Aires: Café de las Ciudades.

COLLIER, D., 1978 - Barriadas y élites, de Odría a Velasco, 170 pp.; Lima: Instituto de Estudios Peruanos (IEP).

CONNOLLY, P., 2013 - La ciudad y el hábitat popular: Paradigma latinoamericano. In: Teorías sobre la ciudad en América Latina (B. Ramírez \& E. Pradilla, comps.), Volumen II: 505-562; México: Universidad Autónoma Metropolitana, Editores e Impresores Profesionales (Edimpro).

DUHAU, E., 1998 - Hábitat popular y política urbana, 304 pp.; México, D. F.: Universidad Autónoma Metropolitana Azcapotzalco.

DUHAU, E., 2001 - La megaciudad en el siglo XXI. De la modernidad inconclusa a la crisis del espacio público. Papeles de Población, 7 (30): 131-161.

GEHL, J., 2013 - La humanización del espacio urbano: la vida social entre los edificios, 215 рp.; Barcelona: Reverté.

HERNÁNDEZ BONILLA, M. , 2008 - Procesos informales del espacio público en el hábitat popular. Revista Bitácora Urbano Territorial, 13 (2): 109-116.

HERZER, H., DI VIRGILIO, M., RODRÍGUEZ, M. C. \& REDONDO, A., 2008 - ilnformalidad o informalidades? Hábitat popular e informalidades urbanas en áreas urbanas consolidadas. Revista Interuniversitaria de Estudios Territoriales, 4 (4): 85-112.

INSTITUTO NACIONAL DE ESTADÍSTICA E INFORMÁTICA (INEI), 2014 - Una Mirada a Lima Metropolitana, 79 pp.; Lima.

JARAMILLO, S., 2013 - Acerca de la investigación en mercados de tierra urbana en América Latina, 22 pp.; Bogotá: Universidad de los Andes, Facultad de Economía, Centro de Estudios sobre Desarrollo Económico (CEDE).

KAHATT, S., 2017 - Estrategias para habitar la pendiente. In: La ciudad de las laderas: Workshop Limápolis 2016 (L. Rodríguez \& P. Muñoz, eds.): 63-73; Lima: Pontificia Universidad Católica del Perú (PUCP).

LUDEÑA, W., 2016 - La ciudad/ladera popular como memoria histórica. Paradojas y persistencia. In: La ciudad de las laderas: Workshop Limápolis 2016 (L. Rodríguez \& P. Muñoz, eds.): 79-89; Lima: Pontificia Universidad Católica del Perú.

MEJÍA CARRIÓN, W., 2009 - Urb. Mariscal Cáceres: De la pampa desértica a la sobrepoblación. Disponible en https://es.scribd.com/document/39902271/Desarrollo -Historico-de-Mariscal-Caceres-Wilmer-Mejia-Carrion (consulta realizada en marzo de 2015). 
MUÑOZ, P. \& RODRÍGUEZ, L., 2015 - Vivienda en laderas. Una política urbana/pública en la periferia de Lima (parte 2). Disponible en http://www2.ual.es/RedURBS/BlogURBS/ vivienda-en-laderas-parte-2/ (consulta realizada el 15 de noviembre de 2017).

PEREYRA, O., 2006 - Forma urbana y segregación residencial en Lima. Debates en Sociología, 31: 69-106.

RIOFRÍO, G., 2004 - Pobreza y desarrollo urbano en el Perú. In: Hoy, las ciudades en el Perú: 73-111; Lima: Centro de Estudios y Promoción del Desarrollo (Desco).

RIOFRÍO, G. \& DRIANT, J.-C., 1987 - ¿Qué vivienda han construido? Nuevos problemas en viejas barriadas, 161 pp.; Lima: Centro de Investigación, Desarrollo y Asesoría Poblacional (CIDAP), Instituto Francés de Estudios Andinos, Asociación de Publicaciones Educativas (TAREA).

RODRÍGUEZ, L., 2016 - La ciudad de las laderas. Estudio y propuesta de vivienda social para las laderas de Lima Metropolitana. Ministerio de Vivienda, Construcción y Saneamiento del Perú (Informe no publicado).

RODRÍGUEZ, L. \& MUÑOZ, P., 2017a - La ciudad de las laderas: Workshop Limápolis 2016, 380 pp.; Lima: Fondo Editorial de la Pontificia Universidad Católica del Perú (PUCP).

RODRÍGUEZ, L. \& MUÑOZ, P., 2017b - La ciudad de las laderas. In: La ciudad de las laderas: Workshop Limápolis 2016 (L. Rodríguez \& P. Muñoz, eds.): 139-147; Lima: Pontificia Universidad Católica del Perú (PUCP).

ROY, A., 2005 - Urban Informality: Toward an Epistemology of Planning. Journal of the American Planning Association, 71 (2): 147-158.

TAKANO, G. \& TOKESHI, J., 2007 - Espacio público en la ciudad popular: reflexiones y experiencias desde el Sur, 80 pp.; Lima: Centro Estudio y Promoción del Desarrollo (DESCO).

VELARDE HERZ, F., 2017 - El espacio público popular. Formas de producción y apropiación de los espacios públicos en la Ciudadela Mariscal Cáceres; Quito: FLACSO Ecuador. Tesis de maestría.

VELARDE, F. \& VÁSQUEZ, J., 2017 - El riesgo en ladera como contingencia urbana. Reflexiones sobre la medición del riesgo y las formas de habitar la ladera. In: Otro urbanismo para Lima. Más allá del mejoramiento de barrios (J. Molnárová, L. Rodríguez Rivero, Á. Espinoza \& R. Fort, eds.): 110-129; Lima: Pontificia Universidad Católica del Perú (PUCP), Universidad Científica del Sur y Grupo de Análisis para el Desarrollo (GRADE). 